\title{
Transition Temperature of Josephson Junction Arrays with Long-Range Interaction
}

\author{
H. R. Shea and M. Tinkham \\ Department of Physics and Division of Engineering and Applied Sciences, Harvard University, Cambridge, Massachusetts 02138
}

(Received 17 June 1997)

\begin{abstract}
We report measurements of the dependence on magnetic field and array size of the resistive transition of Josephson junction arrays with long-range interaction. Because every wire in these arrays has a large number of nearest neighbors ( 9 or 18 in our case), a mean-field theory should provide an excellent description of this system. Our data agree well with this mean-field calculation, which predicts that $T_{c}$ (the temperature below which the array exhibits macroscopic phase coherence) shows very strong commensurability effects and scales with array size. [S0031-9007(97)04071-4]
\end{abstract}

PACS numbers: $74.50 .+\mathrm{r}$

We report an experimental investigation of ordered Josephson junction arrays with long-range interaction (ALRI), of the sort originally proposed in the disordered limit by Vinokur et al. [1]. Although such arrays had been fabricated by Sohn et al. [2], the samples used in the present Letter for the first time have low enough critical currents and hence low enough screening to be in the regime well described by existing theoretical models $[3,4]$.

These arrays consist of two perpendicular sets of $N$ parallel superconducting wires, coupled by Josephson junctions at every point of crossing (see Fig. 1). In this geometry, any horizontal (vertical) wire is nearest neighbor to all vertical (horizontal) wires, and nextnearest neighbor to all other horizontal (vertical) wires. Hence we term the interaction long range. The number of nearest neighbors in these arrays is equal to the array size $N$. This is in sharp contrast to standard (shortrange interaction) $2 \mathrm{D}$ arrays where the number of nearest neighbors (typically 4 or 6 ) is independent of array size.

Arrays with long-range interaction were first proposed by Vinokur et al. [1] as a physical realization of the Sherrington-Kirkpatrick (SK) model [5], which is an analytically studied model of a spin-glass. The SK model assumes the interaction between spins does not depend on the separation between the spins, and therefore does not describe most experimentally studied spin-glass systems. Vinokur et al. showed that for the case where the wires are positionally disordered and a sufficiently strong perpendicular magnetic field is applied, ALRI are very similar to the SK model and admit an analytic solution. More recently, Chandra et al. [4] have shown that even for an ordered array, glassy behavior is expected in a very weak field (less than one flux quantum per row).

The equivalent of "spins" in these ALRI are the phases of the superconducting wires, which are well defined in any given gauge. Since field screening is negligible, the actual field equals the applied field, and we can make the gauge choice $\mathbf{A}=f x \Phi_{0} / a^{2} \hat{\mathbf{y}}$, where $a$ is the lattice constant and $f$ is the flux per cell divided by a flux quantum. In the appropriate limit where the junction critical currents are negligible compared to the wire critical currents, we can write $\mathbf{J}=0$, where $\mathbf{J}$ is the current density in the wires. $\mathbf{J}$ is given by the GinzburgLandau expression

$$
\mathbf{J}=\frac{e}{m}|\Psi|^{2}\left(\hbar \nabla \varphi-\frac{2 e}{c} \mathbf{A}\right),
$$

where $\Psi$ is the order parameter of the wires. With our gauge choice, setting $\mathbf{J}$ to zero implies that the phases $\varphi^{V}$ of the vertical wires are position dependent with $\nabla \varphi^{V}=$ $2 \pi f x / a^{2} \hat{\mathbf{y}}$, while the phases $\varphi^{H}$ of the horizontal wires are constant. Above the transition temperature $T_{c}$, the phases of the wires are uncorrelated. However, when the array is cooled below $T_{c}$, a transition to a macroscopically phase coherent state is predicted to occur.

For an ordered array with long-range interaction in the limit of negligible screening, Sohn et al. [3] have performed a mean-field analysis and computed the transition temperature $T_{c}^{\mathrm{MF}}(f)$ as a function of the applied field and array size. Because each wire has a large number of nearest neighbors, a mean-field theory using the phase of each wire as a classical thermodynamic variable should provide a good description of this system. At zero field they find $T_{c}=N E_{J}\left(T=T_{c}\right) / 2 k_{B}$, where $E_{J}(T)=\hbar i_{c}(T) / 2 e$ and $i_{c}(T)$ is the (unfluctuated) critical current of a single junction at temperature $T$. Note the unusual result that $T_{c}$ should scale with the size of the array. To keep $T_{c}$ of the array well below $T_{c}^{\text {wire }}$, one requires $N \hbar i_{c}^{0} \ll 4 e k_{B} T_{c}^{\text {wire }}$, where $i_{c}^{0}$ is $i_{c}(T=0)$. The computations of Refs. [1], [3], and [4] only hold in the limit of negligible screening, where

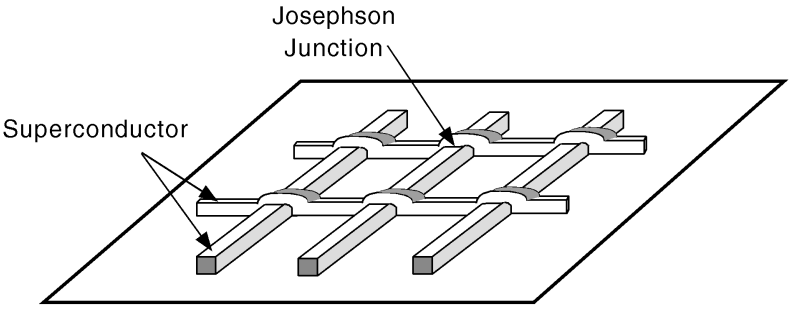

FIG. 1. Schematic drawing of a 2 wire by 3 wire array with long-range interaction. There are Josephson junctions at every crossing point of the superconducting wires. 
the array as a whole screens much less than one flux quantum $\Phi_{0}$, and in the limit when phase gradients along the wires due to current flow are much less than phase drops at the junctions. The former condition can be written as $N^{2} L_{g} i_{c}^{0} \ll \Phi_{0}$, where $L_{g}$ is the geometric inductance of a cell in the array. The latter condition can be expressed as $i_{c}^{\text {junction }} \ll i_{c}^{\text {wire }}$. These three inequalities place very strong limits on the magnitude of $i_{c}^{0}$ for given $N$.

In the experimental work of Sohn and co-workers, $N \hbar i_{c}^{0} / 4 e k_{B} T_{c}^{\text {wire }} \approx 300$ and $N^{2} L_{g} i_{c}^{0} / \Phi_{0} \approx 10^{3}$. Hence their samples were not in the regime defined by the abovementioned theories. We present here the first measurements of ALRI with critical currents small enough (of order $5 \mathrm{nA}$ ) to be in the limit of extremely weak screening, and to have an array $T_{c}$ well below the wire critical temperature. Our data show impressive agreement with the mean-field theory, including extremely strong commensurability effects.

The samples consist of $0.25 \mu \mathrm{m}$ wide $\mathrm{Al}$ wires $\left(T_{c}^{\text {wire }} \approx\right.$ $1.7 \mathrm{~K}$ ) connected by $\mathrm{Al}-\mathrm{AlO}_{x}$-Al junctions, fabricated as follows. A gridlike pattern of lattice constant $2 \mu \mathrm{m}$ is defined using electron-beam lithography on a $\mathrm{Si}$ wafer coated with polymethyl methacrylate (PMMA). A threeangle shadow evaporation technique is used to deposit both sets of wires sequentially without breaking vacuum, using only the single lithography step. The evaporations are done at $45^{\circ}$ to the substrate surface, but at different orientations with respect to the patterned channels. $30 \mathrm{~nm}$ of $99.999 \%$ pure $\mathrm{Al}$ are evaporated in the direction of one set of wires (the "horizontal" set). Al accumulates on the substrate only along those horizontal wires because the PMMA shadows the "vertical" wires. $150 \mathrm{mTorr}$ of $\mathrm{O}_{2}$ is bled into the chamber, and an oxygen plasma is ignited for 20 min to grow an $\mathrm{AlO}_{x}$ barrier. After pumping out the $\mathrm{O}_{2}$, the sample is rotated so that the second and third evaporations (30 nm of $\mathrm{Al}$ each) are done in the direction of the "vertical" wires, going "up" for the second evaporation and "down" for the third, to ensure that the vertical lines are continuous where they "climb" over the horizontal wires. A lift-off completes the process. This shadow evaporation technique yields very high quality underdamped junctions which are a major improvement over those from the previous fabrication technique [2].

The typical single junction resistance is $R_{N}^{J J}=70 \mathrm{k} \Omega$ which corresponds [6] to an unfluctuated critical current $i_{c}^{0}$ of $5.6 \mathrm{nA}$, or $E_{J}(T=0) / k_{B}=0.13 \mathrm{~K}$. Junction uniformity, measured from single junctions cofabricated with the arrays, is approximately $\pm 15 \%$. The lead configuration is shown in the inset of Fig. 2. Current is injected in the first wire of one set, and extracted from the last wire of that same set. We report data on two arrays: one consisting of $9 \times 9$ wires $(8 \times 8$ cells $)$ and the other of $18 \times 18$ wires $(17 \times 17$ cells $)$.

The arrays are cooled to $315 \mathrm{mK}$ in a ${ }^{3} \mathrm{He}$ cryostat within a double $\mu$-metal shield which reduces the stray field to less than $50 \mathrm{mG}$. (A field of $5.2 \mathrm{G}$ corresponds to

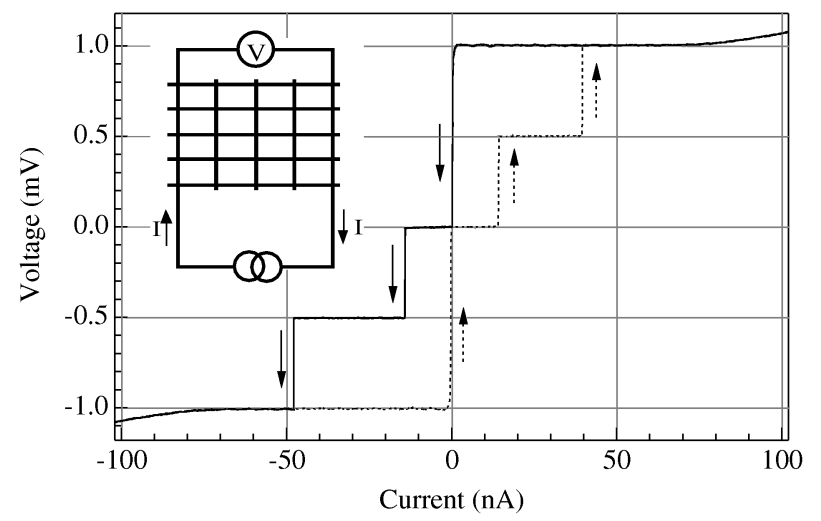

FIG. 2. Voltage-current plot at $0.315 \mathrm{~K}$ in zero field of the $17 \times 17$ cell array. The dashed line corresponds to sweeping current up, the solid line to sweeping current down. There is a finite slope at zero bias, too small to be seen on the graph. The inset is a schematic diagram of the lead configuration used for current injection and voltage measurement.

$f \equiv \Phi_{\text {cell }} / \Phi_{0}=1$ for our $2 \mu \mathrm{m}$ spacing.) Temperature stability is better than $3 \mathrm{mK}$ below $2 \mathrm{~K}$. A small magnetic field is applied perpendicular to the array using a solenoid surrounding the vacuum can of the cryostat. Screening by the array can be neglected because $i_{c}^{0}$ is so small. Quantitatively, the ratio of the maximum flux screened by the array to the flux quantum is much less than one: $N^{2} L_{g} i_{c}^{0} / \Phi_{0} \approx 3 \times 10^{-3} \ll 1$, for $N=18$ and where $L_{g} \approx 4 \mathrm{pH}$ is the geometric inductance of a single cell in the array, modeled as a superconducting square washer [7]. Considerable care was taken to ensure that the arrays are well shielded from $\mathrm{rf}$ and microwave radiation by the use of a shielded room, room-temperature low-pass LC П filters, cold resistors, and cold microwave filters [8].

The current-voltage $(I-V)$ curves for single junctions cofabricated with the arrays do not show a well-defined critical current at $0.3 \mathrm{~K}$ because $E_{J}<k_{B} T$, and hence a finite resistance is observed for all bias currents. The arrays, on the other hand, consisting of many junctions in parallel, do show, at least at the lowest temperatures, a well-defined critical current and strong hysteresis, as expected from underdamped junctions. Figure 2 shows an $I-V$ curve for the $17 \times 17$ array at $f=0$. The two jumps in voltage to $2 \Delta / e$ and $4 \Delta / e$ (where $\Delta$ is the superconducting gap) correspond to all the junctions connected to one, then the other, of the current injection wires going normal. The unfluctuated zero-temperature array critical currents $I_{c}^{0}=N i_{c}^{0}(\sim 60 \mathrm{nA}$ for the $9 \times 9$ wire array, $\sim 100 \mathrm{nA}$ for the $18 \times 18$ wire array) are so small that the measured $I_{c}$ will be significantly less than $I_{c}^{0}$ due to thermal fluctuations. The measured $I_{c}$ actually corresponds to a jump from a finite-voltage (of order $1 \mu \mathrm{V})$ phase-diffusion branch [9] to $2 \Delta / e$ at a current value which is affected by damping as well as $E_{J}$ and $T$.

We therefore focus instead on the differential resistance $R_{d}=d V / d I$ (at zero dc bias) as a function of field and 
temperature since it should be a better measure of the zerocurrent phase coupling of the array (and hence $T_{c}$ ). $R_{d}$ is measured using a PAR 124 lock-in amplifier at $15.6 \mathrm{~Hz}$ with an excitation current of $0.2 \mathrm{nA}$ (corresponding to $\sim I_{c} / 10$ ). Figure 3 shows $R_{d}$ vs $f \equiv \Phi_{\text {cell }} / \Phi_{0}$ plots for several temperatures from 0.4 to $1.7 \mathrm{~K}$ for the $17 \times 17$ cell sample. The curves are not offset. Because $R_{d}(f)$ is periodic in $f$ and symmetric around $f=1 / 2$, we only plot $R_{d}(f)$ for $f$ ranging from 0 to $1 / 2$. $R_{d}(f)$ displays minima at all commensurate fields where $f=$ $p / q, p$ and $q$ being integers smaller than $N$. The $f=$ $1 / 17$ and $f=1 / 16$ states are not clearly resolved but all other commensurate states are clearly present (such as, for instance, all other multiples of $1 / 17$, like $2 / 17$ and $3 / 17$ ). All the measured positions of the resistance minima are within less than $10^{-4} \times \Phi_{0}$ from the ideal computed commensurate field values. We observe very similar behavior for the $8 \times 8$ cell array, with resistance minima at $f=1 / 8,1 / 7, \ldots$ It is a characteristic feature of ALRI that such strong and detailed structure in the $R_{d}(f)$ curve is visible. Standard 2D arrays do not exhibit such richness of structure because they do not have the long-range order needed to support a stable vortex superlattice with such a large lattice constant (e.g., 17 cells).

The deepest resistance minima occur at the most strongly commensurate states: $f=0,1 / 2,1 / 3,1 / 4$. The shape of the $R_{d}(f)$ curve is very similar near all of these states (see Fig. 3). The full widths of the resistance dips (i.e., the field intervals between local maxima on either side of the dips) scale as $1 / q$, with $q=1,2,3 \ldots$ Near integer $f$ (e.g., $f=0,1,2 \ldots$ ) where $q=1$, the resistance increases smoothly from $f=n$ until $f=n \pm[1 /(N-1)]$, i.e., the first adjacent commensurate state, giving a modulation-free half-width

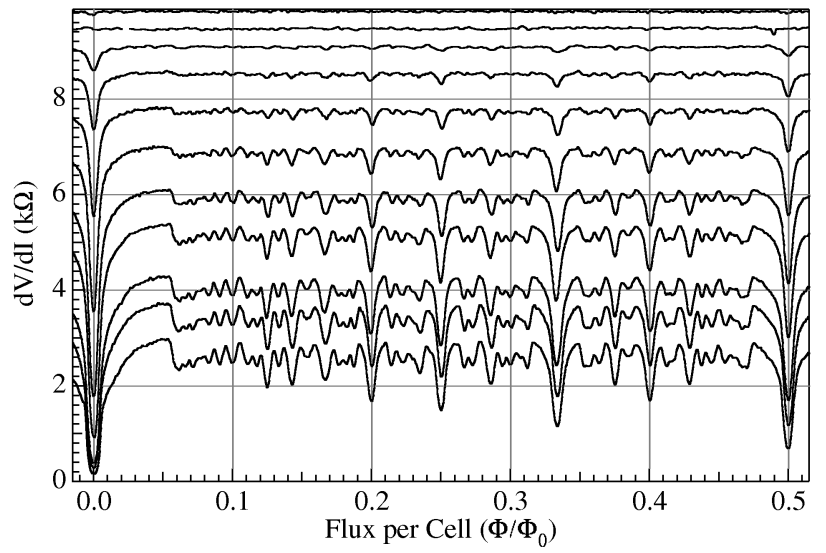

FIG. 3. Plot of zero-bias differential resistance of the $17 \times 17$ cell array vs normalized flux $f$, measured with a $0.2 \mathrm{nA}$ ac excitation, for selected temperatures. The curves are not offset. From the lowest to the highest curve, the temperatures are $0.417,0.702,0.797,0.959,1.047,1.13,1.212,1.288,1.39$, 1.51 , and $1.69 \mathrm{~K}$. The local minima in resistance occur within $10^{-4} \times \Phi_{0}$ of all the commensurate flux values, i.e., at all $f=p / q$ where $p$ and $q$ are integers between 1 and 17 . of $1 /(N-1)$. Corresponding behavior occurs near other strongly commensurate states.

$R_{d}(f)$ was measured for 20 temperatures between 0.315 and $1.8 \mathrm{~K}$, of which 11 are shown in Fig. 3. As the temperature is increased, $R_{d}(f)$ increases and the relative amplitudes of the resistance oscillations decrease until at higher temperatures (but with the wires still superconducting) $R_{d}(f)$ saturates at the normal state resistance of the array $R_{N}^{\text {array }}=2 R_{N}^{J J} / 18$. In order to extract $T_{c}(f)$ from the $R_{d}(f)$ vs $T$ data, we make use of the finite width of the resistive transition. We define the experimental $T_{c}$ using a resistive criterion; for each field value, $R_{d}$ is plotted vs $T$, and $T_{c}$ is taken to be the temperature at which $R_{d}$ interpolates to $\epsilon R_{N}$, and $\epsilon$ is a number between 0 and 1 . Automating this process produces the top two curves of Fig. 4 of $T_{c}(f)$ for $\epsilon=0.5$ (top curve) and $\epsilon=0.375$ (middle curve). For values of $\epsilon$ between approximately 0.4 and 0.8 , the inferred $T_{c}$ scales almost linearly with $\epsilon$.

The bottom curve is the result of a mean-field calculation of $T_{c}^{\mathrm{MF}}(f)$ for a $17 \times 17$ cell array. $T_{c}^{\mathrm{MF}}(f)$ is the temperature above which the order parameter $\eta_{i} \equiv\left\langle e^{i \varphi_{i}}\right\rangle$ is equal to 0 , where $\varphi_{i}$ is the phase of the $i$ th wire and the brackets denote a thermal average. There are no free parameters in this calculation, which consists of using an efficient scheme to find the largest eigenvalue of a $17 \times 17$ matrix given by Eq. (19) of Ref. [3] for each of 1000 field values shown. The eigenvalue problem is solved assuming a temperature-independent $E_{J}$, and $T_{c}^{\mathrm{MF}}$ is finally corrected to account for $E_{J}(T)$, which varies by $\sim 30 \%$ over the temperature range of interest.

The data and mean-field theory curves are in good agreement, both for the $17 \times 17$ cell array in Fig. 4, and for the $8 \times 8$ cell array (not shown). The maxima in the experimental $T_{c}(f)$ obviously occur at commensurate

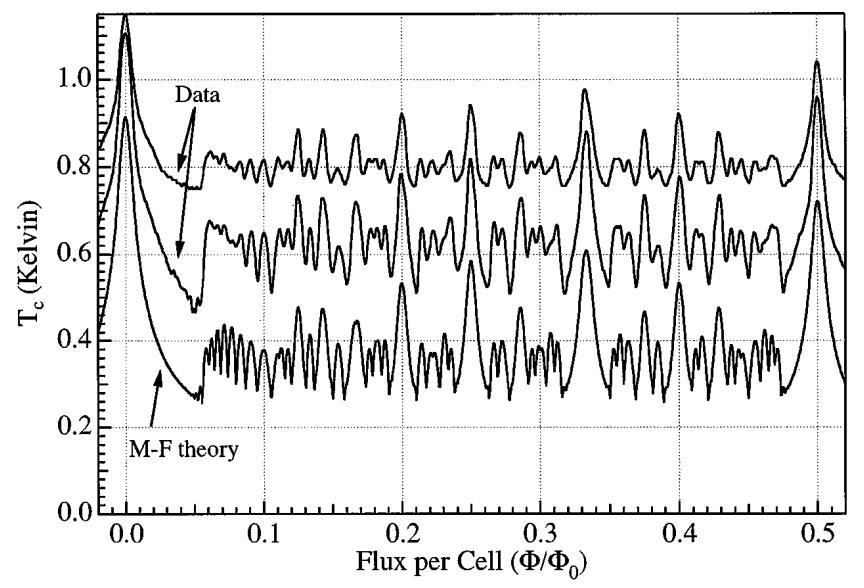

FIG. 4. Plot of the temperature $T_{c}$ corresponding to the onset of macroscopic phase coherence vs normalized flux $f$ for the $17 \times 17$ cell array. The top two curves (data) are computed from the differential resistance vs field data by using a resistive transition criterion for $T_{c}$ of $0.5 R_{N}^{\text {array }}$ (top curve) and $0.375 R_{N}^{\text {array }}$ (middle curve). The lower curve is the result of a mean-field calculation of $T_{c}^{\mathrm{MF}}(f)$. 
fields to the same high accuracy as the minima in the resistive data do, since the critical temperature was extracted from the $R_{d}(f)$ curves. The mean-field theory also predicts local maxima in $T_{c}^{\mathrm{MF}}$ at all commensurate fields: we find that the positions of the clearly discernible maxima in the experimental $T_{c}(f)$ and $T_{c}^{\mathrm{MF}}$ agree to better than one part in $10^{4}$. As can be seen in Fig. 4, the lower resistance criterion gives better quantitative agreement with the mean-field theory $T_{c}^{\mathrm{MF}}$, which is always below the experimental $T_{c}$ (and is defined slightly differently). We cannot use a resistive criterion of less than $\epsilon \approx 0.37$ over the whole field range because at low temperatures, for $f \neq 0$, the array resistance saturates at a nonzero value (up to $\approx 3 \mathrm{k} \Omega$ for incommensurate $f$ ), probably due to macroscopic quantum tunneling of the phases.

In order to compare the experimental $T_{c}$ 's of the $18 \times$ 18 wire and $9 \times 9$ wire arrays, we must first account for the temperature dependence of $E_{J}$ in order to obtain $T_{c}^{*}$, the transition temperature one would observe if $E_{J}$ were constant and the same for both arrays. For $f=0$ we then obtain $T_{c, 18}^{*} / T_{c, 9}^{*}=1.9$, using $\epsilon=0.375$ to determine $T_{c}$ for both arrays. This is very close to the theoretical value of $18 / 9=2$, indicating that $T_{c}$ does indeed scale with system size $N$.

Having verified the mean-field prediction for $T_{c}(f)$, we now briefly discuss the ground-state phase configuration of the wires at zero temperature. In the absence of screening we can write the following simple expressions for the phases of each wire at $x=j a$ and $y=k a$ at zero applied current:

$$
\begin{gathered}
\varphi_{k}^{H}=\varphi_{0}^{H}+2 \pi f N k, \\
\varphi_{j}^{V}=\varphi_{0}^{V}+2 \pi f k j .
\end{gathered}
$$

$\varphi_{k}^{H}$ is the phase of the $k$ th horizontal wire (constant along the wire) and $\varphi_{j}^{V}$ is the phase of the $j$ th vertical wire (depends linearly on the position $y$ along the wire). The only free parameter is $\Delta \varphi_{0}^{H V}=\varphi_{0}^{H}-\varphi_{0}^{V}$. The system energy $E$ is

$$
E=-\sum_{k, j=0}^{N-1} \cos \left(\varphi_{k}^{H}-\varphi_{j}^{V}\right) .
$$

The ground-state energy is found by minimizing $E$ numerically as a function of $\Delta \varphi_{0}^{H V}$ for each field. Once $\Delta \varphi_{0}^{H V}$ is found, all the phase differences are then determined. The local extrema of both $-E_{\min }(f)$ and $T_{c}^{\mathrm{MF}}(f)$ occur at exactly the same fields, with very similar relative amplitudes, indicating that the above simple expressions for the phases of the wires do indeed describe the phases very accurately.

It is very difficult to probe the glassiness of this system using transport measurements because the phases unlock as soon as a small transport current is applied. Even though the arrays are biased well below $I_{c}$, a finite voltage develops across the system because of phase diffusion.
Phase diffusion is unavoidable in the small (i.e., lowcapacitance) and weak junctions required to conform to the model conditions. Since the phases are evolving as $\langle d \varphi / d t\rangle=2 e V_{\mathrm{dc}} / \hbar$, they cannot lock. Hence individual metastable states, the presence of which would confirm the presence of a glass, cannot be probed using our transport technique. For instance, we observe the same $I_{c}$ at every field cool, while trapping into different metastable states should give a range of measured critical currents. Similarly, the $T_{c}$ we measure reflects an average over many configurations and thus reveals very little about the glassiness of the array.

In conclusion, we have fabricated Josephson junction arrays with long-range interaction and extremely weak critical currents. A mean-field theory provides an excellent description of this system because every wire has a large number of nearest neighbors ( 9 or 18 for the arrays presented here). Our data for $T_{c}(f, N)$ are in very good agreement with the mean-field calculation: we find that $T_{c}(f=0)$ scales with system size and observe very strong commensurability effects. The array differential resistance at zero dc bias exhibits minima at all commensurate fields, displaying far more complex, but well understood, structure than standard 2D arrays or wire networks.

We wish to thank R. J. Fitzgerald and M. A. Itzler for their extensive assistance with the measurements and analysis, and J.M. Hergenrother, D. Davidović, and M. S. Rzchowski for their insightful comments. H.R.S. acknowledges financial support from NSERC of Canada and FCAR du Québec. This work was supported in part by ONR Grant No. N00014-96-1-0108, JSEP Grant No. N00014-89-J-1023, and NSF Grant No. DMR92-07956.

[1] V.M. Vinokur, L. B. Ioffe, A. I. Larkin, and M. V. Feigel'man, Zh. Eksp. Teor. Fiz. 93, 343 (1987) [Sov. Phys. JETP 66, 198 (1987)].

[2] L. L. Sohn, M. T. Tuominen, M. S. Rzchowski, J. U. Free, and M. Tinkham, Phys. Rev. B 47, 975 (1993).

[3] L. L. Sohn, M. S. Rzchowski, J. U. Free, and M. Tinkham, Phys. Rev. B 47, 967 (1993).

[4] P. Chandra, L. B. Ioffe, and D. Sherrington, Phys. Rev. Lett. 75, 713 (1995); P. Chandra, M. V. Feigelman, and L. B. Ioffe, Phys. Rev. Lett. 76, 4805 (1996).

[5] D. Sherrington and S. Kirkpatrick, Phys. Rev. Lett. 35, 1792 (1975); S. Kirkpatrick and D. Sherrington, Phys. Rev. B 17, 4384 (1978).

[6] V. Ambegaokar and A. Baratoff, Phys. Rev. Lett. 10, 486 (1963); 11, 1044(E) (1963).

[7] J. M. Jaycox and M. B. Ketchen, IEEE Trans. Magn. MAG-17, 400 (1981).

[8] J. M. Martinis, M. H. Devoret, and J. Clarke, Phys. Rev. B 35, 4682 (1987).

[9] J. M. Martinis and R. L. Kautz, Phys. Rev. Lett. 63, 1507 (1989); R. L. Kautz and J. M. Martinis, Phys. Rev. B 42, 9903 (1990). 